\title{
Análise da formação dos preços na citricultura paulista
}

\author{
Adriana Ferreira Silva ${ }^{1}$, Geraldo Sant'Ana de Camargo Barros ${ }^{2} \&$ Margarete Boteon $^{3}$
}

\section{RESUMO}

Os principais agentes da cadeia da laranja no Brasil não operam em igualdade de poder de mercado. Dessa forma, os preços deixam de refletir somente as condições potenciais de oferta e demanda, mas também a estratégia do segmento com maior poder de mercado. O objetivo deste estudo foi analisar a relação entre o preço do suco de laranja estabelecido no mercado externo (nos negócios entre exportadores e engarrafadores nos países de destino) e o preço da laranja recebido pelos produtores no mercado brasileiro. Este estudo abrangeu a análise estatística/econométrica dos preços do suco de diferentes mercados, de forma a identificar a série (ou séries) de preços mais consistente em explicar o processo de formação dos preços aos produtores, nas safras de 1995/96 a 2012/13. Os resultados indicaram que os preços da laranja são vinculados estruturalmente aos do suco no atacado internacional, independente da fonte considerada, e essa relação pode ser usada em um novo rearranjo negocial entre produtor de laranja e a indústria do suco. Desse confronto, resultará a definição das margens de remuneração industrial e dos produtores rurais. O objetivo é que o novo rearranjo leve a ajustes mais rápidos e proporcionais entre os agentes, e com isso, a relações mais estáveis e transparentes.

Termos de indexação: suco de laranja, transmissão de preços, assimetria.

\section{Analysis of the formation of prices in the citrus industry in São Paulo}

\section{SUMMARY}

The main agents of the orange chain in Brazil do not operate on equal market power. Thus, prices no longer reflect only the potential supply and demand conditions, but also the strategy of the segment with greater market power.This study aims to analyze the relationship between orange juice prices established in international markets (in business between exporters and bottlers in countries of destination) and the orange fruit prices paid to Brazilian farmers. This study involved the statistical and econometric analysis of juice prices from different markets in order to identify the series (or series) more consistent in explaining the price formation for, in the harvests from $1995 / 96$ to $2012 / 13$. Results indicate that the price of orange fruit is fundamentally linked to the price of its juice, regardless of data source considered for testing, and this relationship can be used

\footnotetext{
${ }^{1}$ Universidade Federal de Goiás - UFG, Goiânia, GO, Brasil

${ }^{2}$ Departamento de Economia Administração e Sociologia, Escola Superior de Agricultura Luiz de Queiroz - ESALQ, Universidade de São Paulo - USP, Piracicaba, SP, Brasil

${ }^{3}$ Centro de Estudos Avançados em Economia Aplicada - CEPEA, Escola Superior de Agricultura Luiz de Queiroz - ESALQ, Universidade de São Paulo - USP, Piracicaba, SP, Brasil

Autor correspondente: Adriana Ferreira Silva, Universidade Federal de Goiás - UFG, Rua Samambaia, s/n, Campus Samambaia, CP 131, CEP 74690-900, Goiânia, GO, Brasil. E-mail: adsilva@ufg.br
} 
in a new negotiation rearrangement between orange producer and the juice industry. From this comparison will result in the definition of industrial remuneration margins and farmers producers. The goal is that the new rearrangement will lead to faster and more proportionate adjustments between agents, and with that, to more stable and transparent relationships.

Index terms: orange juice, price transmission, asymmetry.

\section{INTRODUÇÃO}

A cadeia da laranja, como amplamente reconhecido, e à semelhança das mais importantes cadeias agropecuárias brasileiras, é constituída por um segmento agrícola composto por numeroso, mas não homogêneo (por tamanho e tecnologia), contingente de produtores, ladeado a jusante por um número relativamente pequeno de indústrias processadoras ${ }^{1}$. Não se trata, portanto, de uma cadeia em que os principais agentes operem em igualdade de poder de mercado. Nesse contexto, os preços deixam de refletir somente as condições potenciais de oferta e demanda, mas também a estratégia do segmento com maior poder de mercado.

Essa desigualdade de barganha tem gerado conflitos entre produtores e indústria, já relatados em outros trabalhos. Em 1995, um Termo de Cessação de Conduta Anticoncorrencial, determinado pelo Conselho Administrativo de Defesa Econômica (Cade), foi assinado pelas empresas processadoras, sendo estabelecido o fim do contrato padrão. Em 2004, a Polícia Federal fez uma operação de apreensão de documentos, conhecida como Operação Fanta, recolhendo supostas provas de que o conluio entre empresas processadoras para fixação de preços ao produtor de laranja não havia se extinguido (Paulillo \& Almeida, 2009). Em 2011 e 2012, fracassaram as negociações entre representantes da citricultura e da indústria com o objetivo de formar o Conselho dos Produtores de Laranja e das Indústrias de Suco de Laranja (Consecitrus), evidenciando que os conflitos ainda permaneciam (Figueiredo et al., 2013).

De forma geral, as relações estruturais de preços em qualquer cadeia tendem a refletir os fundamentos e a estrutura do mercado - caracterizando uma forma de equilíbrio estrutural, que pode não ter o sentido usual de igualdade entre oferta e demanda por depender também da estrutura do mercado. Se essa estrutura não for concorrencial, como na cadeia citrícola, não se pode falar que oferta e

\footnotetext{
${ }^{1}$ A montante há um número também reduzido de indústrias fornecedoras de insumos, o que tende a contribuir para maiores custos agrícolas. Esta questão não está no foco deste estudo.
}

demanda estejam determinando os preços, mas, sim, que estes estão sendo determinados pelo controle da oferta do produto final (neste caso, suco) e da demanda pela matéria prima (laranja, aqui tratado) ${ }^{2}$.

Entre os fundamentos de mercado podem-se se elencar: comportamento da demanda dos importadores, a tendência da produção e da produtividade e seus reflexos nos custos industriais, de exportação e de produção agrícola. A estrutura de mercado influencia como esses fundamentos vão afetar o preço e o abastecimento do mercado. A estrutura envolve o número e tamanho das empresas, bem como o grau de interdependência nas decisões das mesmas e a forma como essa interdependência é tratada do ponto de vista legal. Podem alterar a estrutura a entrada ou saída de empresas, ampliações e reduções de plantas e suas capacidades produtivas, fusões e aquisições. Em geral é difícil atribuir a um ou mais desses fatores as mudanças observadas.

Neste contexto, a falta de plena concorrência entre as partes de uma cadeia produtiva, como ocorre na cadeia citrícola, pode resultar em menor produção, menores preços da matéria prima e maior preço aos consumidores. Uma reformatação negocial da cadeia visando ao equilíbrio na capacidade de negociação entre os segmentos pode contribuir para maior eficiência econômica e mais adequada e justa distribuição da renda nela gerada. Ao examinar as relações entre os preços do suco e da laranja, este estudo pretende subsidiar os formuladores dessa possível reorganização.

O objetivo geral deste estudo foi analisar a relação entre o preço do suco de laranja estabelecido no mercado externo - nos negócios entre exportadores e engarrafadores nos países de destino - e o preço da laranja recebido pelos produtores no mercado brasileiro - envolvendo o segmento industrial/exportador e o segmento primário (citricultores), contribuindo na geração de novas evidências que favoreçam relações menos conflituosas entre os agentes da cadeia citrícola.

\footnotetext{
${ }^{2}$ Não é objetivo deste estudo a relação entre exportadores e importadores no mercado de destino do suco.
} 


\section{MATERIAL E MÉTODOS}

\section{Fonte e definição das variáveis}

$\mathrm{Na}$ análise dos preços da laranja foram considerados os preços mensais postos na indústria através do mercado spot, que se referem a valores negociados pela indústria paulista com os produtores sem ou através de contratos, porém ambos com fechamento somente para uma safra. Estes dados foram obtidos junto ao Centro de Estudos Avançados em Economia Aplicada da Universidade de São Paulo (CEPEA/USP). Essa série de preços representa principalmente produtores de pequena e média escala que não conseguem comercializar sua produção através de contratos de longo prazo (mais de uma safra), como os grandes grupos de produtores. Os contratos de longo prazo, na sua maioria, já são atrelados aos preços internacionais, enquanto os preços da laranja no mercado spot são fixos na série histórica analisada.

No mercado atacadista internacional de suco, foram analisados dados mensais das seguintes fontes: (a) Foodnews: preço do suco de laranja (FCOJ) posto na Europa; (b) Secretaria de Comércio Exterior (Secex): preço FOB calculado pela relação entre o valor (US\$) e volume exportado para Europa (FCOJ e NFC) e para os Estados Unidos; (c) Bolsa de Nova Iorque (NY - ICE): Cotação futura do suco de laranja (FCOJ) na Bolsa de NY. (média mensal de todos os vencimentos).

O período analisado considerou as safras de 1995/96 a 2012/2013. A escolha do início da série deve-se a nova formatação do mecanismo de fixação de preços, após a extinção do contrato padrão.

\section{Modelo econométrico}

O estudo das relações entre os preços da laranja posta na indústria brasileira e do suco de laranja baseou-se na análise econômica e econométrica de transmissão de preços. Tal análise pode ser realizada, pelo menos, sob três ângulos: (a) relações de causalidade: que preço antecipa em termos temporais os demais; (b) relações estruturais (mais duradouras) de preços: ligados não somente à oferta e demanda, mas também ao poder dominante que fixa margens de longo prazo; e, (c) relações conjunturais: ancoram-se nas relações estruturais, mas podem divergir delas conforme a conjuntura.

Sob diferentes níveis de mercado, a elasticidade de transmissão de preços mede o impacto percentual sobre os preços de um nível (variável dependente) diante um choque (variação) de preços em outro nível de mercado. Se a elasticidade de transmissão medida for igual a um, o choque inicial nos preços é transmitido na mesma proporção no outro nível de mercado. Impacto e choques são proporcionais. Uma elasticidade maior do que um (menor do que um) significa que o impacto é mais do que proporcional (menos que proporcional) ao choque inicial.

Conforme Barros (2012) é preciso destacar que mesmo em mercados concorrenciais, as variações de preços no nível da matéria prima tendem a ser maiores do que aquelas ao nível do processamento ou atacado. Uma das razões para tal é o fato de os custos industriais (salários, energia, combustíveis, etc.) serem mais estáveis do que o preço as matéria-prima. A literatura especializada não dá indicações quanto ao efeito de estruturas não concorrenciais sobre os padrões de transmissão de preços, podendo ocorrer que a transmissão seja até mais intensa nesses casos (Barros, 2012).

Paralelamente ao conhecimento do processo de transmissão de preços, também será verificada a possível existência de assimetria de transmissão de preços, ou seja, a existência de diferença na intensidade da transmissão quando da queda ou elevação de preços. Conforme Peltzman (2000) e Meyer \& Cramon-Taubadel (2004), a teoria econômica tem pouco, ou talvez nada, a dizer sobre a questão de assimetria embora a maioria dos estudos apontem para sua existência e relacionem o fato à falta de concorrência ${ }^{3}$. Cramon-Taubadel \& Meyer (2001) apontam evidências de que métodos normalmente utilizados para testar assimetria tendem a resultados que apontam excessivamente para a existência de assimetria ${ }^{4}$. Isso não quer dizer que se deva negligenciar a assimetria de preços: ao contrário, qualquer que seja sua causa, ela deve ser combatida caso esteja prejudicando uma das partes numa transação.

\footnotetext{
${ }^{3}$ Peltzman (2000), constata que "the notion that some weakness of competition underlies price asymmetries is commonly mentioned [...] However, I find that attributing asymmetries to imperfect competition is unlikely to be rewarding" (p. 468). Meyer e Cramon-Taubadel (2004), concluem que: "Hence it is not clear a priori whether market power will lead to positive or negative asymmetry" (p. 4).

${ }^{4}$ Cramon-Taubadel e Meyer (2001), argumentam da seguinte forma: "The obvious implication of our preliminary analysis is, however, that the results of tests for asymmetric price transmission must be interpreted with great caution if there is reason to suspect that there are structural breaks in the price series being investigated. Since it appears reasonable to assume that there are structural breaks in many real price series, our results imply that the very common finding of asymmetric price transmission in empirical applications in agriculture and elsewhere may be more artefact than fact" (p. 12).
} 
Visando a analisar tais relações, empregou-se um conjunto métodos estatísticos/econométricos, os quais são resumidos abaixo, conforme sua etapa de realização:

$1^{\text {a }}$ Etapa) Análise da relação de causalidade entre as séries: suco versus laranja e suco versus suco, para dados de diferentes fontes. Para tal realizou-se o teste de causalidade de Granger (1969), que mede se mudanças em uma variável contém informação sobre mudanças futuras em outra variável. Este teste baseia-se em duas premissas, a saber: a) a previsibilidade é um conceito análogo ao de causalidade e b) a relação entre causa e efeito é temporária no sentido de que este não pode surgir antes daquela.

Na reinterpretação de Hoover (2001), citado por Matos (2003), uma variável X causa Y no sentido de Granger, se a história passada de Y não oculta inteiramente o efeito de $\mathrm{X}$ sobre $\mathrm{Y}$. Isso, na prática, significa que $\mathrm{X}$ causa ou precede $Y$, se a variância do erro da regressão de Y sobre seus próprios valores passados e os valores passados de $\mathrm{X}$ for estatisticamente menor do que a variância do erro da regressão de Y sobre seus valores passados somente.

Para diferentes níveis de mercado, as relações de causalidade indicam se mudanças de preço num nível de mercado antecedem mudanças em outro nível. Elas indicam, portanto, que o preço num determinado nível de mercado é um indicador do que ainda vai se passar num outro nível.

É preciso deixar claro que a causalidade pode ocorrer sob as seguintes circunstâncias: (a) num mercado concorrencial, qualquer nível de mercado pode iniciar uma mudança de preço sempre que houver alteração nas suas condições de oferta e demanda; posteriormente a alteração é passada a outro nível de mercado a jusante ou montante; (b) num mercado não-concorrencial, um nível de mercado (indústria, por exemplo) pode alterar inicialmente seu preço de compra (laranja) em antecipação a mudanças nas condições de mercado onde vende seu produto processado, cujo preço pode ou não influenciar; (c) o fato de um nível se antecipar a outros não implica necessariamente que esse nível seja o formador de preço.

A análise da causalidade envolve duas etapas principais. Primeiramente, deve-se realizar a análise de raiz unitária ${ }^{5}$ e na sequência, comprovando-se que as séries não são estacionárias e integradas de mesma ordem deve-se realizar o teste de co-integração (Engle \& Granger, 1987).

$2^{\text {a }}$ Etapa) Conhecido o sentido de causalidade e quais séries "causam" o preço da laranja spot, parte-se para

\footnotetext{
${ }^{5}$ Os procedimentos adotados para os testes de raiz unitária podem ser vistos em Dickey e Fuller (1981); Phillips e Perron (1988); Dickey e Pantula (1987).
}

o estudo de longo prazo das transmissões de preços. Esta análise visa investigar a existência de relações estáveis, e de longo prazo, entre as variáveis consideradas, mesmo perante desvios de curto prazo, que afastem as séries entre si.

Não se trata de um estado de equilíbrio, no sentido de ter-se oferta igual à demanda, mas, sim de uma relação de preços que reflete em boa parte as decisões do segmento mais concentrado diante da evolução do consumo, da produção (num quadro de incertezas de custos na cadeia produtiva, de clima e de ocorrência de pragas e doenças nas lavouras). Essa relação serve como um guia para os ajustes de preço observados no mercado.

A modelagem adotada nesta etapa tomou como base a análise de cointegração, por meio do procedimento de Johansen (1988). Nesta análise, o objetivo é verificar a existência ou não de relações estáveis e de longo prazo entre os preços, utilizando um modelo Vetorial de Correção de Erro (VEC), e a decomposição do erro da variância para verificar a direção e a intensidade dos choques entre os preços.

O método de Johansen (1988) consiste na utilização de estimadores de máxima verossimilhança para testar a presença e estimar vetores de cointegração. Este procedimento está centrado na relação existente entre o rank (ou posto) de uma matriz e suas raízes características.

Enders (1995) resume a relação de cointegração em três modelos: mesma ordem de integração de todas as variáveis cointegradas; combinação linear estacionária de variáveis não estacionárias; e o número de vetores de cointegração existentes é igual à quantidade de variáveis do modelo menos um.

Desta forma, o primeiro passo na análise de cointegração consiste em verificar a estacionariedade e a ordem de integração das séries. A partir de então, realiza-se o teste de Johansen (1988).

$3^{\circ}$ Etapa) Na última etapa testou-se a presença de assimetria nas transmissões de preços entre os preços ao produtor e ao atacado. A presença de assimetria é detectada pela ocorrência de duas diferentes elasticidades de transmissão: uma para aumento e outra para queda de preços. Este teste foi realizado através do modelo de Houck (1977), o qual tem como função identificar se acrescimentos ou decréscimos de preços são transmitidos com a mesma intensidade. Sua descrição detalhada pode ser encontrada em diversos trabalhos como: Aguiar (1993), Mayorga et al. (2007) e Figueiredo et al. (2013). 


\section{RESULTADOS E DISCUSSÃO}

\section{Relações de causalidade entre as séries}

O teste de causalidade foi conduzido com as variáveis em logaritmo e na diferença, dada a não estacionariedade das séries. Como esperado, a análise dos testes de raiz unitária, para todas as séries, indicou a presença de raiz unitária. Sendo integradas de ordem um I(1), foi necessária a aplicação de uma diferença para torná-las estacionárias.

Para a análise de causalidade, o número de defasagens inseridas no modelo foi resultado da análise do correlograma dos resíduos e dos menores valores dos critérios de informação Akaike e Schawrz (AIC), e Hannan-Quinn (HQ).

Analisando-se os resultados do teste de causalidade de Granger entre a série de preços da laranja e os preços do suco, para as diferentes fontes (Tabela 1), conclui-se que se deve rejeitar a hipótese nula de não causalidade de NY para a laranja, enquanto no sentido contrário, a hipótese nula deve ser rejeitada. O mesmo resultado foi observado no modelo em que se considera a relação entre os preços da laranja e os preços do suco, com fonte na FoodNews, indicando causalidade unidirecional, com o preço ao atacado (agora, FoodNews) causando os preços da laranja.

Já no modelo em que se considera a relação dos preços da Secex com os preços da laranja, não se rejeitou a hipótese nula em ambos os sentidos. Desta forma, não foi identificada a causalidade de Granger dos preços do suco com a série de preços da laranja, e vice-versa.
A partir destes resultados pode-se concluir que os preços do suco, com fonte tanto na bolsa de NY, quanto com fonte na FoodNews, interferem (precedem) a formação dos preços na laranja, permitindo-se fazer inferências a respeito do processo de formação dos preços ao nível do produtor.

Observando-se os resultados do teste de causalidade de Granger, entre os preços do suco de laranja, para as três fontes consideradas (Tabela 2), rejeita-se, portanto, a hipótese nula de não causalidade de NY para Secex; logo, os preços NY causam (ou antecedem) os preços da Secex. No sentido contrário, (Secex para NY), a hipótese nula é rejeitada, assim Secex não causa NY.

A causalidade unilateral também foi constatada na relação entre os preços da FoodNews e Secex. Pelo teste de Granger, rejeita-se a hipótese nula de não causalidade no sentido da FoodNews para os preços com fonte na Secex. Já no sentido contrário, não rejeitou-se a hipótese nula de que Secex não Granger causa FoodNews, ou seja, os preços da Secex não precedem, temporalmente, os preços da FoodNews, embora o contrário seja verdadeiro.

Os resultados do teste de causalidade também apontaram para a causalidade de NY para FoodNews. Em sentido contrário, FoodNews não causa NY, não se rejeitou a hipótese nula.

A Figura 1 sintetiza, esquematicamente, o resultado geral do teste de causalidade. As setas indicam o sentido da causalidade, ou seja, a relação entre as séries quando há precedência temporal entre elas.

As informações contidas na Figura 1 revelam a importância dos preços da bolsa de NY na precedência temporal de todos os demais preços de suco e também da laranja, no período

Tabela 1. Teste de causalidade de Granger entre os preços da laranja e do suco

\begin{tabular}{lcccc}
\hline \multicolumn{1}{c}{ Hipótese Nula $\left(\mathbf{H}_{\mathbf{0}}\right)$} & Defasagem & Teste F & V. C. (5\%) & Decisão \\
\hline NY e LARANJA & & & & \\
NY não granger causa LARANJA & 1 & 3,84013 & 3,920 & ${\text { Rejeita } \mathrm{H}_{0}}$ \\
LARANJA não granger causa NY & 1 & 1,04864 & 3,920 & ${\text { Não Rejeita } \mathrm{H}_{0}}$ \\
FOODNEWS e LARANJA & & & & \\
FOOD não granger causa LARANJA & 1 & 0,71770 & 3,920 & ${\text { Rejeita } \mathrm{H}_{0}}$ \\
LARANJA não granger causa FOOD & 1 & 3,37743 & 3,920 & ${\text { Não Rejeita } \mathrm{H}_{0}}^{\text {SECEX e LARANJA }}$ \\
SECEX não granger causa LARANJA & 1 & & & \\
LARANJA não granger causa SECEX & 1 & 0,29537 & 3,92 & Não Rejeita $\mathrm{H}_{0}$ \\
\hline
\end{tabular}

Fonte: análise dos autores. 
Tabela 2. Resultados do teste de causalidade de Granger entre os preços do suco

\begin{tabular}{|c|c|c|c|c|}
\hline Hipótese Nula & Def. & Teste F & V.C. $(5 \%)$ & Decisão \\
\hline \multicolumn{5}{|l|}{ NY e SECEX } \\
\hline NY não granger causa SECEX & 2 & 3,53902 & 3,072 & Rejeita $\mathrm{H}_{0}$ \\
\hline SECEX não granger causa NY & 2 & 0,81247 & 3,072 & Não Rejeita $\mathrm{H}_{0}$ \\
\hline \multicolumn{5}{|l|}{ FOOD e SECEX } \\
\hline FOOD não granger causa SECEX & 3 & 6,67866 & 2,696 & Rejeita $\mathrm{H}_{0}$ \\
\hline SECEX não granger causa FOOD & 3 & 0,48157 & 2,696 & Não Rejeita $\mathrm{H}_{0}$ \\
\hline \multicolumn{5}{|l|}{ FOOD e NY } \\
\hline FOOD não granger causa NY & 3 & 1,30836 & 2,696 & Não Rejeita $\mathrm{H}_{0}$ \\
\hline NY não granger causa FOOD & 3 & 2,78575 & 2,696 & Rejeita $\mathrm{H}_{0}$ \\
\hline
\end{tabular}

Fonte: análise dos autores.
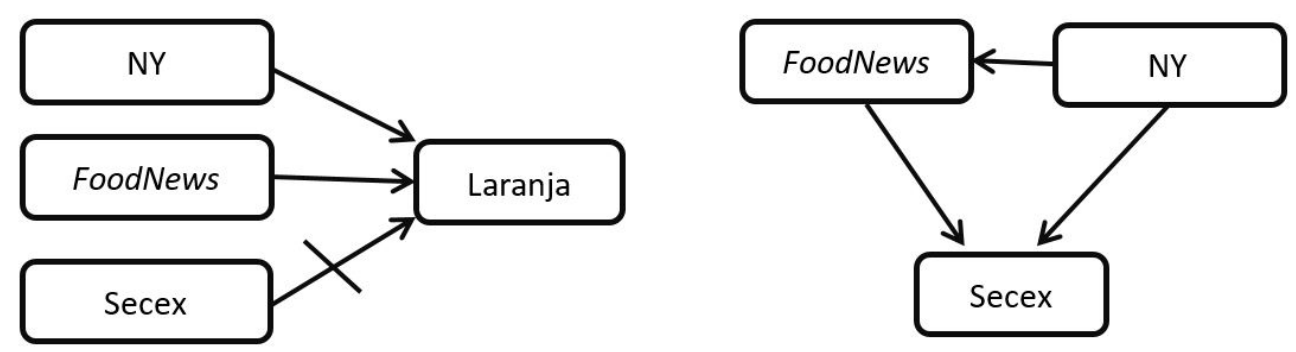

Figura 1. Relações de causalidade entre preços do suco e os preços da laranja - NY = bolsa de Nova Iorque e Secex $=$ Secretaria de Comércio Exterior (Fonte: Resultados da pesquisa).

analisado. Estes preços, assim como a série da FoodNews, geram impacto sobre os preços da laranja, e também sobre os preços da Secex. Esta última fonte foi a única a não preceder a formação dos preços da laranja, no intervalo considerado, daí a representação da seta com um corte.

Portanto, pode-se afirmar que, no tocante ao potencial referencial para o Brasil, o preço informado pela FoodNews tem para o caso brasileiro vantagens consideráveis, pois, além de referir-se ao mercado europeu (foco da oferta brasileira), reflete bem o preço informado pela bolsa de NY, a qual, apesar de sua influência em escala global, guarda vinculação estreita com a oferta e a demanda nos Estados Unidos e, em parte, com a oferta brasileira.

\section{Relações de curto e longo prazo}

Conhecido o sentido de causalidade e quais séries "causam" o preço da laranja spot, partiu-se para o estudo do equilíbrio do mercado, também referido como de longo prazo. Esse equilíbrio reflete uma determinada estrutura do mercado. Em geral sendo concentrada em nível da indústria e relativamente pulverizada do lado dos produtores rurais e consumidores é de se esperar que a relação de preços tenha o preço industrial como (neste caso, do suco) causador das variações de preço da laranja.

Não se trata de um estado de equilíbrio, no sentido de ter-se oferta igual à demanda, mas, sim de uma relação de preços estruturais que reflete em boa parte as decisões do segmento mais concentrado diante da evolução dos fundamentos do mercado, tais como consumo, da produção (num quadro de incertezas de custos na cadeia produtiva, de clima e de ocorrência de pragas e doenças nas lavouras), etc. Essa relação serve como um guia para os ajustes de preço observados no mercado.

Em qualquer instante que se considere, o mercado acha-se numa situação de desequilíbrio, com preços se ajustando ao longo do tempo no sentido de alcançar o equilíbrio. Daí que a análise da transmissão de preços do suco para a fruta trata inicialmente do estabelecimento 
do equilíbrio estrutural, seguido da movimentação de preços em direção a esse equilíbrio. Nesse processo de ajuste é possível que o próprio preço de equilíbrio se altere, afetando o caminho a ser seguido pelos preços de mercado.

Os resultados da relação de equilíbrio de longo prazo são apresentados na Tabela 3. Nesta etapa, a série de preços do suco da Secex não foi empregada, uma vez que pelo teste de Granger, rejeitou-se a hipótese de causalidade com os preços da laranja. No mais, sendo as séries não estacionárias, o teste de cointegração de Johansen (1988) foi realizado com as séries na primeira diferença.

A presença de pelo menos $r$ vetores de cointegração foi testada a partir dos resultados da estatística traço, e a ordem do modelo (número de defasagens inseridas) foi definida com base na análise do correlograma dos resíduos e dos menores valores dos critérios de informação (AIC, BIC, HQ).

A hipótese nula $(r=0)$ de não haver cointegração entre as séries de preços de laranja e do suco, para qualquer fonte (bolsa de NY ou FoodNews), foi rejeitada. Assim, em ambos os casos, os resultados da estatística traço ( $\lambda$ traço) permitiram concluir pela existência de um vetor de cointegração ao nível de significância de $5 \%$.

Identificada a existência de vetores de cointegração em um número inferior ao número de variáveis, formulou-se um modelo Vetorial de Correção de Erro - VEC. Desta forma, em cada estimativa do VAR, foi necessário incluir os respectivos vetores de correção de erro, permitindo inferir sobre a velocidade em que as variáveis convergem para uma situação de equilíbrio de longo prazo. Além disso, feita a constatação da integração de primeira ordem das séries, e a modelagem do VAR foi realizada com todas as séries na primeira diferença.

O resultado da equação de longo prazo entre os preços (utilizando os respectivos vetores de correção de erro), para a análise da formação do preço spot da laranja em relação ao preço do suco, é resumido abaixo com as variáveis na forma logarítmica:
Para os preços do suco com fonte na FoodNews, tem-se: $L_{t}=1,035 F_{t}+\ldots+\mu_{t}$ $(7,602)$

Para os preços do suco com fonte na bolsa de NY, tem-se:

$L_{t}=1,062 N Y_{t}+\ldots+\mu_{t}$

$(5,684)$

em que: $L_{t}=\log$ do preço da laranja spot posta na indústria brasileira no mês t; $F_{t}=\log$ do preço do suco FoodNews no mês t; $N Y_{t}=\log$ do preço do suco na bolsa de NY no mês t.

Os resultados encontrados evidenciam a sensibilidade dos preços de equilíbrio da laranja aos preços de equilíbrio do suco (medido por diferentes fontes), uma vez que a elasticidade de longo prazo encontrada pelo método de cointegração foi igual ou pouco maior (mas não significantemente diferente) do que a unidade e com o sinal positivo. Nesse sentido, no equilíbrio, as variações no preço do suco no mercado internacional são, em média, transmitidas proporcionalmente para o preço da laranja no mercado interno.

Para melhor avaliar o papel de cada preço internacional, calculou-se a decomposição da variância dos erros de previsão dos preços mensais da laranja. Observa-se que as movimentações de preço de laranja de um mês para outro, em relação aos preços do suco FoodNews é basicamente um processo de ajuste do próprio preço da laranja ( $89 \%$ da variância) com pequena influência do preço do suco (11\%): ou seja, a movimentação mensal de preços da laranja não sofre novos impactos importantes do preço internacional do suco (Tabela 4).

Quando se considera os preços de NY, ocorre comportamento do mesmo tipo, notando-se, todavia, que no processo de ajustamento do preço da laranja ainda há impactos relativamente importantes (10 a $21 \%)$ de mudanças no preço do.

Estes últimos resultados - cointegração e decomposição de variância - indicam que grande parte das variações mensais do preço da laranja são ajustes de curto prazo a

Tabela 3. Resultados do Teste de cointegração de Johansen sobre as séries de preços

\begin{tabular}{cccccc}
\hline \multirow{2}{*}{ Variáveis } & Hipótese & & Hipótese & \multirow{2}{*}{$\boldsymbol{\lambda}$ traço } & \multicolumn{1}{c}{ Valores } \\
\cline { 2 - 3 } \cline { 5 - 5 } & Nula & Alternativa & & críticos 5\% \\
\hline Laranja & $\mathrm{r} \leq 1$ & $\mathrm{r}>1$ & 1,952 & 3,841 \\
Suco FoodNews & $\mathrm{r}=0$ & $\mathrm{r}>0$ & $\mathrm{r}>1$ & 21,999 & 15,408 \\
Laranja & $\mathrm{r} \leq 1$ & $\mathrm{r}>0$ & 2.472 & 3,841 \\
Suco NY & $\mathrm{r}=0$ & & 20,414 & 15,408 \\
\hline
\end{tabular}

Fonte: Resultados da pesquisa. 
Tabela 4. Decomposição da variância dos erros de previsão (\%) dos preços da Laranja

\begin{tabular}{ccccc}
\hline Mês & Laranja & FoodNews & Laranja & NY \\
\hline 1 & 99,77 & 0,23 & 89,77 & 10,23 \\
5 & 89,87 & 11,23 & 79,27 & 20,73 \\
10 & 89,88 & 11,22 & 79,26 & 20,74 \\
\hline
\end{tabular}

Fonte: Resultados da Pesquisa.

Tabela 5. Resumo dos resultados do teste de Assimetria

\begin{tabular}{cccc}
\hline & \multicolumn{3}{c}{ Efeito após dois meses } \\
\cline { 2 - 4 } & Acréscimo & Decréscimo & Teste $\mathbf{F}$ \\
\hline FoodNews & 0,595 & 0,699 & 2,279 \\
Bolsa NY & 1,097 & 1,712 & 3,994 \\
\hline
\end{tabular}

Fonte: cálculo dos autores.

Tabela 6. Frequência de elasticidades de transmissão de preços

\begin{tabular}{cccccc}
\hline & \multicolumn{2}{c}{ Choques FoodNews } & & \multicolumn{2}{c}{ Choques NY } \\
\cline { 2 - 3 } \cline { 5 - 6 } & positivos & negativos & & positivos & negativos \\
\hline Elasticidade & $(\%)$ & $(\%)$ & & $(\%)$ & $(\%)$ \\
$>1$ & 56 & 57 & & 33 & 35 \\
0 a 1 & 22 & 14 & & 27 & 23 \\
$<1$ & 22 & 29 & & 40 & 42 \\
\hline
\end{tabular}

Fonte: cálculo dos autores.

uma situação de equilíbrio, com limitada influência do preço internacional no decorrer do processo. Isso não quer dizer que o preço internacional não seja relevante; ao contrário, ele é fundamental para determinar a direção para a qual o preço mensal da laranja vai caminhar, como se viu na discussão acima sobre a relação de equilíbrio entre os preços da fruta e do suco.

\section{Assimetria na transmissão dos preços}

De acordo com os resultados da análise econométrica de assimetria entre os preços ao produtor e os preços internacionais, após dois meses (quando se completa a transmissão) via modelo de Houck (1977), nota-se que há diferença entre a transmissão de acréscimos e decréscimos de mudanças nos preços do suco para os preços da laranja (Tabela 5). As quedas do suco, para ambas as fontes (FoodNews e NY), são transmitidas aos preços da laranja com maior intensidade num período de dois meses. Caso se considere a FoodNews como fonte de dados, uma queda de $10 \%$ nos preços do suco levaria a uma queda de $6,99 \%$ no preço da laranja. A variação vai para $17,12 \%$ no caso de Nova York. No caso de um aumento de preço do suco, as transmissões para os preços da laranja passam a 5,95\% e 10,97\%, respectivamente.

Na Tabela 6, são apresentadas as frequências de casos na amostra correspondentes a três intervalos de elasticidades de transmissão: (i) maior que a unidade, o que significa que a variação de preço do suco foi transmitida mais do que proporcionalmente e no mesmo sentido para o preço da laranja; (ii) entre zero e a unidade, neste caso a variação foi transmitida menos do que proporcionalmente e no mesmo sentido; (iii) menor do que zero, o que implica que a variação no suco foi transmitida em sentido contrário para o preço da laranja.

No caso dos choques com origem no suco com fonte na FoodNews, constata-se que as transmissões no mesmo sentido, e mais do que proporcionais predominaram em $57 \%$ dos meses. Já as transmissões em sentido contrário (elasticidades negativas) ocorreram em 29\% dos casos, e as transmissões de mesmo sentido e menos do que proporcionais (elasticidades entre zero e um) ocorreram em torno de $14 \%$ da amostra.

De forma geral, pode-se concluir que as transmissões mensais de preço, independente da fonte dos preços do suco, apontam para volatilidade e pouca transparência do 
mercado, seja provocando inversões na movimentação de preços, seja exagerando as variações ocorridas.

\section{CONCLUSÕES}

Este estudo tratou da formação do preço da laranja pago aos produtores brasileiros pelas indústrias, segmento cuja concentração confere, em tese, poder de mercado para fixação desse preço.

No que respeita à relação entre variações de preços de suco e laranja, constatou-se haver uma parte estrutural e outra conjuntural. A parte estrutural resulta da soma dos efeitos dos fundamentos (oferta e demanda) mais o efeito do poder de mercado detido pela indústria. No aspecto estrutural, os resultados da pesquisa apontaram que o preço da laranja se vincula fundamentalmente ao preço do suco, qualquer que seja o seu indicador. A bolsa de NY destacou-se como principal sinalizador de preços internacionais do suco de laranja. A fonte FoodNews, por se referir ao mercado europeu, para onde se destina predominantemente o suco brasileiro e manter forte vinculação contemporânea antecipada com os preços de NY, também despontou como um bom indicador de referência do preço da laranja.

As evidências apontaram a existência de relação causal de longo prazo entre as séries de preço do suco (independente da fonte) e dos preços da laranja ao produtor. Mesmo tendo influência de longo prazo, os indicadores de preços do suco, independente da fonte, pouco afetaram a formação dos preços de curto prazo da laranja.

Assim, as variações de preços estruturais do suco tendem ser repassadas aos produtores de laranja. Entretanto, o repasse completo pode demorar um ano ou mais. Como abreviar essa transmissão é uma questão que mereceria reflexões adicionais.

No tocante à assimetria na transmissão das variações dos preços do suco para os da laranja, foram encontradas evidências favoráveis a essa hipótese, de forma que as transmissões de altas de preço de suco chegam ao produtor da laranja em proporções menores do que quando se transmitem quedas de preço.

Conforme já discutido, a assimetria de ajustes de preço carece de fundamentação teórica adequada e não se relaciona necessariamente ao uso de poder de mercado. Mesmo assim, sua detecção é importante, visto que pode prejudicar um dos lados do mercado em consideração.
Um exame detalhado dos dados disponíveis indica que: (a) as mudanças de preços se dão em direções que ora favorecem o produtor de laranja, ora favorecem a indústria, sem uma clara vantagem para uma das partes; (b) em termos de intensidade de variação, observa-se que elas são predominantemente maiores no caso do preço spot da laranja, ou seja, este preço é bem mais volátil.

Para um novo arranjo negocial, é importante uma avaliação do custo industrial e dos custos dos produtores de laranja. Desse confronto, resultarão os termos para o rearranjo negocial entre produtor de laranja e a indústria para definição dessa margem, remunerando à mesma taxa as duas partes da cadeia produtiva, e levando a ajustes mais rápidos e proporcionais entre os preços de suco e laranja.

\section{REFERÊNCIAS}

Aguiar, D. R. D. (1993). A questão da transmissão de preços agrícolas. Revista de Economia e Sociologia Rural, 31(4), 291-308.

Barros, G. S. C. (2012). Economia da comercialização agrícola. Piracicaba: CEPEA. Recuperado em 10 de nov. 2016, de http://www.cepea.esalq.usp.br/pdf/1_economia comercializacao_agricola.pdf

Cramon-Taubadel, S. V., \& Meyer, J. (2001). Asymmetric price transmission: fact or artefact? In Proceedings of The 71th EAAE Seminar The food consumer in the early 21st century. Zaragoza: Diputation general de Aragon. Recuperado em 27 de set. de 2016, de http://www. jochenmeyer.de/pdf/cramon-meyer.pdf

Dickey, D. A., \& Fuller, W. A. (1981). Likelihood ratio statistics for auto-regressive time series with a unit root. Econometrica, 49(4), 1057-1072.

Dickey, D. A., \& Pantula, S. G. (1987). Determining the order of differencing in autoregressive processes. Journal of Business \& Economic Statistics, 5(4), 455-461.

Enders, W. (1995). Applied econometric time series (2nd ed.). United States: John Wiley \& Sons, Inc.

Engle, R. F., \& Granger, C. W. J. (1987). Co-integration and error correction: representation, estimation and testing. Econometrica, 55(2), 251-276.

Figueiredo, A. M., Souza Filho, H. M., \& Paulillo, L. F. O. (2013). Análise das margens e transmissão de preços no Sistema Agroindustrial do suco de laranja 
no Brasil. Revista de Economia e Sociologia Rural, 51(2), 331-350.

Granger, C. W. J. (1969). Investigating causal relations by econometric models and cross spectral methods. Econometrica, 37(3), 424-438.

Houck, J. P. (1977). No approach to specifying and estimating nonreversible functions. American Journal of Agricultural Economics, 59(3), 570-572.

Johansen, S. (1988). Statistical analysis of cointegration vetors. Journal of Economic Dynamic and Control, Amsterdam, 12(3), 231-254.

Matos, O. C. (2003). Inter-relações entre desenvolvimento financeiro, exportações e crescimento econômico: análise da experiência brasileira (Notas Técnicas, 40). Brasília: Banco Central do Brasil.

Mayorga, R. O., Khan, A. S., Mayorga, R. D., Lima, P. V. S., \& Margarido, M. A. (2007). Análise de transmissão de preços do mercado atacadista de melão do Brasil. Revista de Economia e Sociologia Rural, 45(3), 675-704.
Meyer, J., \& Cramon-Taubadel, S. (2004). Asymmetric price transmission: a survey. Journal of Agricultural Economics, 55(3), 581-611.

Paulillo, L. F., \& Almeida, L. M. M. C. (2009). A coordenação agroindustrial citrícola brasileira e os novos recursos de poder: dos políticos aos jurídicos. Organizações Rurais \& Agroindustriais, 11(1), 11-27.

Peltzman, S. (2000). Prices rise faster than they fall. Journal of Political Economy, 108(3), 466-502.

Phillips, P. C. B., \& Perron, P. (1988). Testing for unit roots in time series regression. Biometrika, 75, 335-346.

Recebido: Agosto 15, 2017

Aceito: Outubro 31, 2018

Como citar: Silva A.F., Barros G.S.C., \& Boteon M. (2018). Análise da formação dos preços na citricultura paulista. Citrus Research \& Technology, 39, e1040. https://doi.org/10.4322/crt.14418 